\title{
Comparison of MR Imaging and Dual-Energy CT for the Evaluation of Cartilage Invasion by Laryngeal and Hypopharyngeal Squamous Cell Carcinoma
}

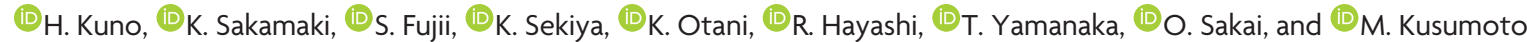

\begin{abstract}
BACKGROUND AND PURPOSE: Dual-energy CT can distinguish iodine-enhanced tumors from nonossified cartilage and has been investigated for evaluating cartilage invasion in patients with laryngeal and hypopharyngeal squamous cell carcinomas. In this study, we compared the diagnostic accuracy of MR imaging and of a combination of weighted-average and iodine overlay dual-energy CT images in detecting cartilage invasion by laryngeal and hypopharyngeal squamous cell carcinomas, in particular thyroid cartilage invasion.
\end{abstract}

MATERIALS AND METHODS: Fifty-five consecutive patients who underwent 3T MR imaging and 128-slice dual-energy CT for preoperative initial staging of laryngeal or hypopharyngeal squamous cell carcinomas were included. Two blinded observers evaluated laryngeal cartilage invasion on MR imaging and dual-energy CT using a combination of weighted-average and iodine-overlay images. Pathologic findings of surgically resected specimens were used as the reference standard for evaluating sensitivity, specificity, and the areas under the receiver operating characteristic curve of both modalities for cartilage invasion by each type of cartilage and for all cartilages together. Sensitivity and specificity were compared using the McNemar test and generalized linear mixed models.

RESULTS: Dual-energy CT showed higher specificity than MR imaging for diagnosing all cartilage together (84\% for MR imaging versus $98 \%$ for dual-energy CT, $P<.004)$ and for thyroid cartilage ( $64 \%$ versus $100 \%, P<.001)$, with a similar average area under the curve $(0.94$ versus $0.95, P=.70)$. The sensitivity did not differ significantly for all cartilages together $(97 \%$ versus $81 \%, P=.16)$ and for thyroid cartilage $(100 \%$ versus $89 \%, P=.50$ ), though there was a trend toward increased sensitivity with MR imaging.

CONCLUSIONS: Dual-energy CT showed higher specificity and acceptable sensitivity in diagnosing laryngeal cartilage invasion compared with MR imaging.

ABBREVIATIONS: AUC = area under the curve; $I O=$ iodine overlay; $R O C=$ receiver operating characteristic; SAFIRE = sinogram-affirmed iterative reconstruction; $\mathrm{SCC}=$ squamous cell carcinoma; WA = weighted-average

n patients with laryngeal and hypopharyngeal squamous cell carcinomas (SCCs), diagnosing cartilage invasion is extremely important for making treatment-related decisions. The thyroid cartilage plays a critical role in primary tumor staging because the

Received February 13, 2017; accepted after revision November 10

From the Departments of Diagnostic Radiology (H.K., K.S., M.K.) and Head and Neck Surgery (R.H.), National Cancer Center Hospital East, Kashiwa, Chiba, Japan; Departments of Radiology (H.K., O.S.), Otolaryngology-Head and Neck Surgery (O.S.), and Radiation Oncology (O.S.), Boston Medical Center, Boston University School of Medicine, Boston, Massachusetts; Department of Biostatistics (K.S., T.Y.) Yokohama City University, Yokohama, Kanagawa, Japan; Division of Pathology (S.F.), Exploratory Oncology Research and Clinical Trial Center, National Cancer Center, Kashiwa, Chiba, Japan; and Advanced Therapies Innovation Department (K.O.), Siemens Healthcare K.K., Shinagawa-ku, Tokyo, Japan.

Please address correspondence to Hirofumi Kuno, MD, PhD, Department of Diagnostic Radiology, National Cancer Center Hospital East, 6-5-1, Kashiwanoha,

Kashiwa, Chiba 277-8577, Japan; e-mail: hkuno@east.ncc.go.jp

Indicates article with supplemental on-line table.

Indicates article with supplemental on-line photo.

http://dx.doi.org/10.3174/ajnr.A5530 extent of thyroid cartilage invasion is a defining factor between T3 and T4a stages. ${ }^{1}$ When tumor extends through the thyroid cartilage into the superficial soft tissue of the neck, the patient is staged T4a and may require total laryngectomy. ${ }^{2-6}$ In cases in which cartilage invasion is localized without transcartilaginous extension, potentially organ-preserving treatment may still be performed. ${ }^{2-5,7,8}$ Therefore, inappropriate treatment choices secondary to over- or underestimation of thyroid cartilage invasion can have grave implications for a patient's quality of life. ${ }^{9}$

MR imaging and CT are used for staging of laryngeal and hypopharyngeal SCCs. However, there is no clear consensus as to which imaging technique is best-suited for evaluating cartilage invasion. Each institution has its own protocol, depending on the availability of imaging equipment and the radiologists' preference and experience. MR imaging has higher soft-tissue contrast resolution and higher sensitivity than conventional CT in diagnosing laryngeal cartilage invasion by laryngeal or hypopharyngeal SCCs. ${ }^{10}$ However, motion artifacts are a serious problem with MR 
imaging in patients with laryngeal or hypopharyngeal cancer with difficulty in breathing and swallowing. ${ }^{11}$ Additionally, edema or inflammatory changes in cartilage may have findings similar or identical to those of tumor invasion on MR imaging. ${ }^{10,12}$ CT has high spatial and temporal resolution and remains the preferred technique for imaging the larynx and hypopharynx. However, nonossified cartilage and tumors may have similar attenuation values, ${ }^{2,13}$ making them almost indistinguishable, especially when the tumor is located adjacent to nonossified thyroid cartilage.

Recently, emerging dual-energy CT techniques have been investigated for head and neck cancer imaging with the potential for improved tumor visualization and characterization. ${ }^{14-21}$ In particular, techniques using iodine overlay (IO) images were found useful for distinguishing iodine-enhanced tumors from nonossified cartilage $\mathrm{e}^{1,14,19,22}$ and for having higher specificity than conventional CT, without a deterioration of sensitivity, in particular for the evaluation of thyroid cartilage invasion. ${ }^{14}$ Furthermore, interobserver agreement is usually poor for conventional CT and was found to be higher for dual-energy CT. ${ }^{14,23}$ These new dualenergy CT techniques have prompted re-evaluations of the diagnostic performance of CT compared with MR imaging in diagnostic fields where MR imaging has been routinely used. ${ }^{24-26}$ The purpose of this study was to compare MR imaging and dualenergy CT in their ability to evaluate cartilage invasion, in particular thyroid cartilage invasion, by laryngeal and hypopharyngeal SCCs.

\section{MATERIALS AND METHODS Patients}

This retrospective study was approved by the institutional review board at National Cancer Center Hospital East. The requirement to obtain written informed consent was waived. Between September 2010 and September 2014, six hundred five consecutive newly diagnosed patients with histologically proved laryngeal or hypopharyngeal SCCs underwent contrast-enhanced dual-energy CT for cancer staging using 128-slice dual-source CT. Among them, 111 patients $(18 \%)$ also underwent 3T MR imaging before treatment. Fifty-three of the 111 patients (48\%) were excluded because they were treated nonsurgically ( 5 underwent radical radiation therapy and 48 received chemoradiotherapy). We excluded an additional 3 patients: Two were unable to complete the MR imaging examination, and another patient's MR imaging study was nondiagnostic secondary to severe motion artifacts. The remaining 55 patients $(50 \%)$ were enrolled in this study. All subjects were treated with surgery (16 patients underwent total laryngectomy, and 39 underwent total pharyngolaryngectomy), and pathologic findings of surgical specimens were used as the reference standard. Of 55 patients, 17 patients (31\%) had laryngeal SCCs (7 supraglottic, 4 glottic, 6 transglottic) and 38 (69\%) had hypopharyngeal SCCs (27 piriform sinus, 4 postcricoid, 7 posterior pharyngeal wall).

\section{MR Imaging Techniques}

All MR imaging studies were performed on a 3T MR imaging system (Achieva TX; Philips Healthcare, Best, the Netherlands) with a 16-channel head and neck coil. Axial turbo spin-echo T1weighted (TR/TE, 559/8.8 ms; flip angle, 90 FOV, 230 mm; ma- trix, $288 \times 288$ ), T2-weighted (TR/TE, 4481/90 ms; flip angle, $90^{\circ}$; FOV, $230 \mathrm{~mm}$; matrix, $320 \times 320$ ), and contrast-enhanced fatsaturated fast-field echo T1-weighted images (TR/TE, 179/3.5 ms; flip angle, $70^{\circ}$; FOV, $230 \mathrm{~mm}$; matrix, $304 \times 304$ ) were obtained parallel to the vocal cords with a 3-mm thickness and 1-mm gap. Additionally, 3D imaging using T2-weighted and pre- and postcontrast-enhanced T1-weighted imaging was also performed from $1.0 \mathrm{~cm}$ above the hyoid bone to the inferior margin of the cricoid cartilage for all patients. 3D T1-weighted images were acquired in the transverse plane using a $3 \mathrm{D}$ turbo-field echo sequence, precontrast (TR/TE, 4.6/2.3 ms; flip angle, $15^{\circ}$; FOV, 230 $\mathrm{mm}$; matrix, $224 \times 224)$ and postcontrast with fat-saturation (TR/TE, 5.9/3.5 ms; flip angle, 15º FOV, $230 \mathrm{~mm}$; matrix, $224 \times$ 224) with a $1-\mathrm{mm}$ thickness and 0 -mm gap.

\section{Dual-Energy CT Techniques}

All dual-energy CT studies were performed with 128-slice dualsource CT scanner (Somatom Definition Flash; Siemens, Erlangen, Germany) in the dual-energy CT mode ${ }^{27}$ with the following parameters: 100 and Sn140-kV tube voltages, 200 and 200 effective $\mathrm{mAs}, 0.33$-second rotation time, $32 \times 0.6-\mathrm{mm}$ collimation with a $\mathrm{z}$-flying focal spot, and a pitch of 0.6. The average CT dose index was $14.45 \mathrm{mGy}$. Patients received $60-150 \mathrm{~mL}$ (600 mg I/kg) of iodinated contrast medium (iohexol 300; Ioverin, Teva Pharma Japan, Nagoya, Japan; or iopamidol 370; Oypalomin, Konica Minolta, Tokyo, Japan) intravenously at a rate of $2.5 \mathrm{~mL} / \mathrm{s}$. The scan started 70 seconds after the start of the injection and proceeded from the base of the skull to the bottom of the neck.

Two image sets $(100 \mathrm{kV}$ and $\mathrm{Sn} 140 \mathrm{kV})$ were reconstructed with $1-\mathrm{mm}$ slice thickness and a $0.7-\mathrm{mm}$ increment with D30f kernels, and a third linearly blended image set (weighted-average [WA] images) with a B30f kernel was used to obtain a $120-\mathrm{kV}$ equivalent image. ${ }^{16} \mathrm{WA}$ images are used as diagnostic images because they are equivalent in terms of image quality to single-energy $120-k V$ CT images. Sinogram-affirmed iterative reconstruction (SAFIRE) was used for WA images (SAFIRE strength 1) and for $100-\mathrm{kV}$ and Sn140-kV images (SAFIRE strength 3). IO images were generated using a 3-material decomposition analysis (syngo Dual Energy, Brain Hemorrhage; Siemens) as proposed in a recent study. ${ }^{14}$ The window for color coding of IO images was visually adjusted to maximize enhancement in tumor tissue and minimize noise in muscle tissue per case. Parallel ranges with 2.0-mm-thick sections and a 16 -cm FOV were generated from the WA images, the virtual unenhanced images, and the IO images as follows: axial and coronal sections parallel and vertical to the vocal cords, respectively (in soft-tissue and bone windows), from 1.0 $\mathrm{cm}$ above the hyoid bone to the inferior margin of the cricoid cartilage.

\section{Image Evaluation}

The MR imaging and dual-energy CT diagnostic criteria for cartilage invasion are defined below (summarized in On-line Table).

MR Imaging. Cartilage invasion was "positive" in cases in which the cartilage displayed signals similar to those of the adjacent tumor on all pre- and postcontrast T1- and T2-weighted images in the cortical bone, cartilage, and bone marrow space. ${ }^{12}$ When the cartilage had higher signal intensity than the adjacent tumor on 
T2-weighted images or when stronger enhancement was present on postcontrast $\mathrm{T} 1$-weighted images, the abnormal signal intensity was diagnosed as negative because these changes may represent reactive changes. ${ }^{12}$ Interpretation always started with the $2 \mathrm{D}$ sequence images, followed by $3 \mathrm{D}$ sequence images when $2 \mathrm{D}$ sequence images alone were insufficient due to artifacts. When the $2 \mathrm{D}$ and $3 \mathrm{D}$ sequence findings were mismatched, the diagnosis obtained on higher quality images was used.

Dual-Energy CT. Image interpretation always started with the WA image to evaluate tumor location, extension, and invasion. ${ }^{14}$ Cartilage with asymmetric sclerosis (ossification) without erosion or lysis was diagnosed as negative on WA images, because these are usually considered reactive changes. ${ }^{28,29}$ When a lesion had been evaluated as having erosion or lysis of ossified cartilage on WA images, the iodine distribution in the remaining nonossified cartilage was evaluated on IO images for a final diagnosis of either positive or negative for cartilage invasion. Areas of ossified cartilage were thoroughly excluded from evaluation on IO images because the dual-energy algorithms could not distinguish calcium from iodine. ${ }^{14,26,30}$

Two radiologists (with 9 and 5 years of experience, respectively, in head and neck radiology), blinded to the clinical history and the image from the other technique, independently analyzed the images. The images were presented in random order in 2 sessions, initially with only MR images, followed 5 weeks later with only dual-energy CT images. The invasion of the thyroid, cricoid, and arytenoid cartilages was evaluated using the following 5-point-scale scoring system: 1 , definitely negative; 2 , probably negative; 3 , erosion (possibly positive); 4 , lysis (probably positive); and 5, transmural extralaryngeal spread through the cartilage (definitely positive). ${ }^{14}$ We arrived at the final diagnosis by consensus. The readers reached consensus in the same manner by discussion when a discrepancy existed for MR imaging and dualenergy CT.

Image-quality in terms of motion artifacts was also evaluated on a 5-point scale: grade 1, nondiagnostic with major artifacts; grade 2, major artifacts with most organs depicted with diagnostic image quality; grade 3 , moderate artifacts with low image quality but diagnostic; grade 4, minor artifacts with good image quality; and grade 5, no artifacts with excellent image quality.

\section{Pathologic Evaluation}

A pathologist (with 22 years of experience) evaluated the surgical specimens, including all cartilage around tumors of all 55 patients. The specimens were fixed in formalin, decalcified, and then sliced horizontally with a thickness of $5 \mathrm{~mm}$ to match the crosssectional MR and dual-energy CT images. The histopathologic findings served as the reference standard.

\section{Statistical Analysis}

The image quality of MR imaging and dual-energy CT was compared by applying the Wilcoxon signed rank test to the imageevaluation grades. For estimating the sensitivity and specificity of both modalities for the detection of cartilage invasion, we used the diagnostic confidence scores with a cutoff point of 3 as a positive diagnosis and generated contingency tables separately for each type of cartilage and for all cartilages together. Because each patient had only 1 thyroid and only 1 cricoid cartilage, we applied the McNemar test to compare sensitivity and specificity for these cartilages. For comparisons of sensitivity and specificity for the evaluation of arytenoid cartilages and of all cartilages together, generalized linear mixed models were used to take into account correlations between multiple cartilages in the same patient. ${ }^{12}$ The overall diagnostic performances were compared using the areas under the curve (AUCs) of the receiver operating characteristic (ROC) curve. For thyroid cartilage, partial AUCs corresponding to a specificity range of $90 \%-100 \%$ were also compared because this specificity range is clinically the most relevant. Interreader agreement between the independent evaluations of the 2 readers for the scores based on the MR imaging and the combination of WA and IO images was estimated by weighted $\kappa$ statistics. We applied weighted $\kappa$ statistics, taking into account that large differences of $>1$ point between the scores of the 2 readers were more serious than smaller differences. ${ }^{31}$ We used commercial software (SAS, Version 9.3 for Windows; SAS Institute, Cary, North Carolina) for the generalized linear mixed modeling and Stata (Version 12.1; StataCorp, College Station, Texas) for all other tests. $P<.05$ indicated a significant difference.

\section{RESULTS}

\section{Image Quality Assessment}

All MR and WA or IO images generated with the dual-energy CT data were considered of diagnostic image quality (grade 2 or more). Motion artifact grading scores are listed in Table 1 . The median image-quality grading score was 3 (interquartile range, 0 ) for MR imaging and 4 (interquartile range, 0 ) for dual-energy CT $(P<.001)$.

\section{Pathologic Findings of Surgically Resected Specimens}

We examined 220 cartilages ( 55 thyroid, 55 cricoid, 110 arytenoid cartilages) of 55 patients. Cartilage invasion was found in 36 of the 220 preparations $(16 \%$; 19 thyroid, 8 cricoid, 9 arytenoid cartilages) and on a per-patient basis in 19 of the 55 patients (35\%). Invasion of the thyroid cartilage was found in all 19 patients whose specimens tested positive for cartilage invasion. Extralaryngeal extension of tumor cells with or without cartilage invasion was confirmed pathologically in 34 of $55(62 \%)$ patients. The 17 laryngeal tumors were classified as pT3 $(n=7)$ and pT4a $(n=$ $10)$, and the 38 hypopharyngeal tumors were classified as pT2 $(n=5)$, pT3 $(n=7)$, and pT4a $(n=26)$.

\section{Relationship between Imaging Findings and Histopathologic Diagnosis of Surgical Specimens}

MR Imaging. Among the 36 cartilage invasions that were histopathologically confirmed, 35 were correctly detected by MR imaging and only one was incorrectly detected as a false-negative finding ( 0 thyroid, 0 cricoid, and 1 arytenoid cartilage). However, MR imaging also led to 29 false-positive findings ( 13 thyroid, 6 cricoid, and 10 arytenoid cartilages) seen in areas of ossified cartilages. Inflammatory changes with desmoplastic reaction were found in many ossified cartilages, especially in areas of fatty marrow with calcification for thyroid cartilage (Fig 1), and mimicked cartilage invasion. In addition, the area of 
Table 1: Summary of motion artifact grading scores for MRI and dual-energy $\mathrm{CT}^{\mathrm{a}}$

\begin{tabular}{|c|c|c|c|c|c|c|c|}
\hline & 1 & 2 & 3 & 4 & 5 & Mean Score (SE) & $P$ Value \\
\hline All patients $(n=55)$ & & & & & & & $<.001^{\mathrm{b}}$ \\
\hline MRI & 0 & 3 & 48 & 4 & 0 & $3.02(0.05)$ & \\
\hline Dual-energy CT & 0 & 0 & 0 & 46 & 9 & $4.16(0.05)$ & \\
\hline T1 or T2 stage $(n=6)$ & & & & & & & .050 \\
\hline MRI & 0 & 0 & 3 & 3 & 0 & $3.50(0.22)$ & \\
\hline Dual-energy СT & 0 & 0 & 0 & 4 & 2 & $4.33(0.21)$ & \\
\hline T3 stage $(n=13)$ & & & & & & & $<.001^{\mathrm{b}}$ \\
\hline MRI & 0 & 1 & 11 & 1 & 0 & $3.00(0.11)$ & \\
\hline Dual-energy CT & 0 & 0 & 0 & 12 & 1 & $4.08(0.08)$ & \\
\hline T4a stage $(n=36)$ & & & & & & & $<.0001^{\mathrm{b}}$ \\
\hline MRI & 0 & 2 & 34 & 0 & 0 & $2.94(0.04)$ & \\
\hline Dual-energy CT & 0 & 0 & 0 & 30 & 6 & $4.17(0.06)$ & \\
\hline
\end{tabular}

Note:-SE indicates standard error.

a Data are number of patients. The rating grade is as follows: grade 1, nondiagnostic with major artifacts; grade 2, major artifacts with most organs depicted with diagnostic image quality; grade 3, moderate artifacts with image quality low but diagnostic; grade 4, minor artifacts with good image quality; grade 5, no artifacts with excellent image quality.

${ }^{b}$ Indicates a significant difference using the Wilcoxon signed rank test $(P<.05)$.

tumor extension in cartilage tended to be overestimated by MR imaging (On-line Figure).

Dual-Energy CT. Dual-energy CT correctly detected 29 cartilage invasions. However, 7 invasions were missed ( 2 thyroid, 2 cricoid, and 3 arytenoid cartilages), among them 3 minor invasions of tumor cells into an ossified part of the cartilage (2 thyroid and 1 cricoid) with an extent of $<3$-mm diameter in the histopathologic specimens (On-line Figure). There were no false-positive findings of thyroid cartilage invasion, but there were 4 false-positive findings of cricoid $(n=1)$ and arytenoid $(n=3)$ cartilage invasion where erosive changes seemed to be present on dual-energy CT images.

\section{Diagnostic Performance of MR Imaging and Dual-Energy CT}

The diagnostic performance of MR imaging and dual-energy CT for patients who underwent surgery is summarized in Table 2.

For analysis of all cartilages together, the specificity of dualenergy CT (98\%; 95\% CI, 95\%-99\%) for the detection of cartilage invasion was significantly higher $(P=.004)$ than that of MR imaging ( $84 \%$; $95 \% \mathrm{CI}, 78 \%-89 \%)$. The point estimate of the sensitivity of dual-energy CT (81\%; 95\% CI, 64\%-92\%) was lower than that of MR imaging (97\%; 95\% CI, 85\%-100\%), but the difference between the sensitivities of the modalities was not statistically significant $(P<.16)$. There was no indication of a true difference in the total AUCs $(0.97 ; 95 \%$ CI, $0.88-1.00$ for MR imaging; and 0.95 ; 95\% CI, $0.89-1.00$ for dual-energy CT; $P=.19)$.

For thyroid cartilage, the specificity of dual-energy CT was significantly higher $(P<.001)$ for the detection of cartilage invasion (100\%; 95\% CI, 90\%-100\%) than MR imaging (64\%; 95\% CI, 46\%-79\%), while no significant differences $(P=.50)$ were found between the sensitivities for the detection of cartilage invasions of MR imaging (100\%; 95\% CI, 82\%-100\%) and dual-energy CT (89\%; 95\% CI, 67\%-99\%). The ROC curves for thyroid cartilage and the corresponding areas under the curve for diagnoses with MR imaging and dual-energy CT are shown in Fig 2. There was no indication of a difference in the mean areas under ROC curve $(0.94 ; 95 \%$ CI, $0.88-1.00$ for MR imaging versus 0.95 ; 95\% CI, $0.89-1.00$ for dual-energy CT; $P=.700$ ) for the evaluation of thyroid cartilage. The calculated partial AUC at the low false-positive ratio area between 0.0 and 0.1 showed that dualenergy CT $(0.09$; 95\% CI, $0.08-1.00)$ was superior to MR imaging $(0.01 ; 95 \% \mathrm{CI}, 0.00-0.63)$ for the evaluation of thyroid cartilage $(P=.003)$.

Analyses of other cartilages showed the following results: No significant differences were found between the sensitivities for the detection of cartilage invasion of MR imaging and dual-energy CT: cricoid cartilage (100\%; 95\% CI, 63\%-100\% for MR imaging versus 75\%; 95\% CI, 35\%-97\% for dual-energy CT) and arytenoid cartilage (89\%; 95\% CI, 52\%-100\% versus $67 \%$; 95\% CI, $30 \%-93 \%$ for dual-energy CT). No significant difference between the specificities of both modalities were found for cricoid cartilage $(P=.06)$ and arytenoid cartilage $(P=.09)$, where the specificities were estimated as $87 \%(95 \% \mathrm{CI}, 74 \%-95 \%)$ and $91 \%(95 \% \mathrm{CI}$, $83 \%-95 \%$ ) for MR imaging compared with $98 \%$ (95\% CI, 89\%$100 \%$ ) and $97 \%$ (95\% CI, 92\%-100\%), respectively, for dualenergy CT. There was no indication of a true difference in the total AUCs for cricoid cartilage $(0.99$; 95\% CI, $0.97-1.00$ for MR imaging versus 0.93 ; $95 \% \mathrm{CI}, 0.85-1.00$ for dual-energy CT; $P=.15$ ) and arytenoid cartilage $(0.95 ; 95 \% \mathrm{CI}, 0.88-1.00$ for MR imaging versus 0.93 ; $95 \% \mathrm{CI}, 0.87-0.99$ for dual-energy CT; $P=.50$ ).

\section{Interreader Agreement}

Interreader agreement of the 2 readers was assessed with quadratic weighted $\kappa$ statistics for MR imaging and for the combination of WA and IO images with the following results ${ }^{31}$ : excellent agreement of 0.80 (95\% CI, 0.70-0.89) and almost perfect agreement of 0.84 (95\% CI, 0.74-0.93), respectively, for the all cartilage together; almost perfect agreement of 0.88 (95\% CI, 0.611.00 ) and 0.90 (95\% CI, 0.63-1.00), respectively, for thyroid cartilage; substantial agreement of 0.69 (95\% CI, 0.45-0.97) and 0.79 (95\% CI, 0.53-1.00), respectively, for cricoid cartilage; and substantial agreement of 0.75 (95\% CI, 0.56-0.93) and 0.77 (95\% CI, 0.59-0.95), respectively, for arytenoid cartilage. These results indicated that both modalities have similar interreader agreement.

\section{DISCUSSION}

In this study, we evaluated the diagnostic performance of MR imaging and the combination of WA and IO dual-energy CT images in the evaluation of cartilage invasion by laryngeal and 

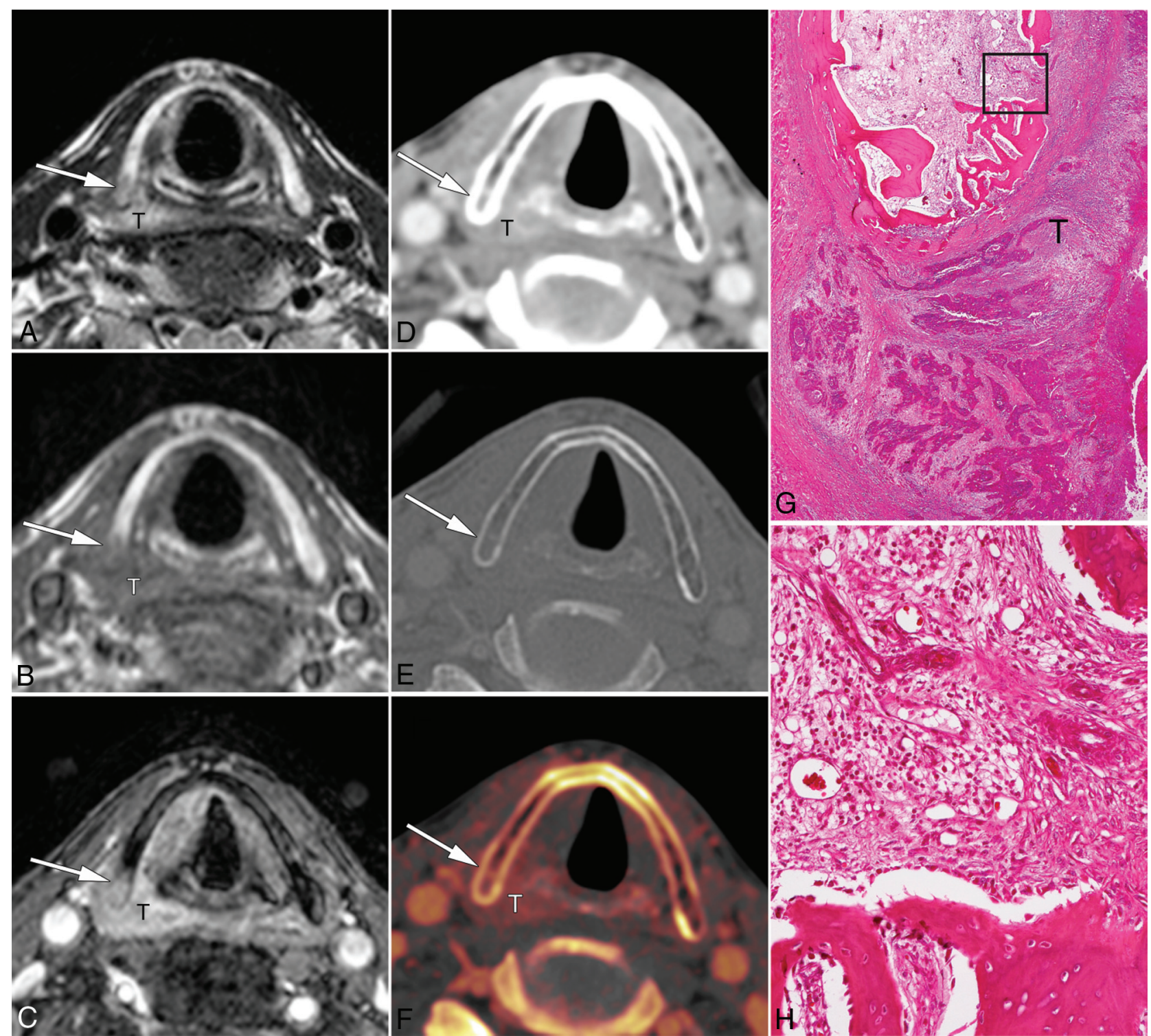

FIG 1. False-positive findings for thyroid cartilage invasion on MR imaging in a 59-year-old man with hypopharyngeal cancer. A, T2-weighted MR image obtained at the glottis level shows a tumor $(T)$ arising from the right piriform sinus with intermediate signal intensity. The adjacent posterior right thyroid lamina also shows an area of intermediate signal intensity (arrow). B, T1-weighted image shows that the tumor (T) has low signal intensity, whereas adjacent thyroid cartilage has similar signal intensity (arrow). C, Contrast-enhanced fat-suppressed Tl-weighted MR image shows similar contrast enhancement of the tumor ( $T$ ) and adjacent thyroid cartilage (arrow). A weighted-average image does not show erosion or lysis at the same level ( $D$, soft-tissue window; $E$, bone window). $F$, lodine overlay image shows enhancement of tumor (T) more clearly and is not used for the diagnosis of cartilage according to the findings of the WA image. G, A micrograph of the corresponding axial slice of the surgical specimen at the same level shows that the squamous cell carcinoma cells do not permeate into the right thyroid cartilage lamina (hematoxylin-eosin stain; original magnification, $\times 5$ ). $H$, Magnified photograph (square in $G$ ) of the posterior part of the right thyroid cartilage lamina with enhancement on MR imaging shows moderate infiltration of lymphocytes into the medullary space, accompanied by fibrosis and aggregation of macrophages, without tumor (H\&E stain; original magnification, $\times 200)$.

hypopharyngeal SCCs. The specificity of dual-energy CT was significantly superior to that of MR imaging when evaluating all cartilages combined and when evaluating thyroid cartilage alone. The point estimates of the sensitivity were higher for MR imaging than for dual-energy CT; however, these differences were not significant for all cartilages combined and for thyroid cartilage alone.

There was also no significant difference in the areas under the ROC curve for the detection of thyroid cartilage invasion of both modalities, suggesting that the overall diagnostic performance might be similar for dual-energy CT and MR imaging and the highest specificities of dual-energy CT may have been achieved in a trade-off against the sensitivity. However, although the total areas under the curve of the ROC curve were identical, the curves showed a crossover at 0.14 and the sensitivity of dual-energy CT at low false-positive ratios ranging between 0.0 and 0.1 was higher than that of MR imaging. ${ }^{32}$ The histopathologic specimens of the false-negative cases (thyroid and cricoid cartilages) seen on dualenergy CT revealed that most had minimal invasion of tumor cells into an ossified part of the cartilage with an extent of $<3-\mathrm{mm}$ diameter, which was not detectable with dual-energy CT, whereas massive cartilage invasion was misdiagnosed in none of the cases as negative findings. In the era of laryngeal conservation, the re- 
Table 2: Relationship between dual-energy CT/MR imaging and histopathologic findings for the detection of cartilage invasion ${ }^{\mathrm{a}}$

\begin{tabular}{|c|c|c|c|c|c|c|c|c|}
\hline & TP & TN & FN & FP & Sensitivity (\%) & Specificity (\%) & PPV (\%) & NPV (\%) \\
\hline \multicolumn{9}{|c|}{ Thyroid cartilage $(n=55)$} \\
\hline MRI & 19 & 23 & 0 & 13 & $100(82-100)$ & 64 (46-79) & $59(41-76)$ & $100(85-100)$ \\
\hline Dual-energy CT & 17 & 36 & 2 & 0 & 89 (67-99) & 100 (90-100) & 100 (80-100) & $95(82-99)$ \\
\hline$P$ value ${ }^{b}$ & & & & & .5 & $<.001$ & & \\
\hline \multicolumn{9}{|c|}{ Cricoid cartilage $(n=55)$} \\
\hline MRI & 8 & 41 & 0 & 6 & $100(63-100)$ & $87(74-95)$ & $57(29-82)$ & $100(91-100)$ \\
\hline Dual-energy CT & 6 & 46 & 2 & 1 & 75 (35-97) & $98(89-100)$ & $86(42-100)$ & $96(86-99)$ \\
\hline$P$ value ${ }^{b}$ & & & & & .5 & .06 & & \\
\hline \multicolumn{9}{|c|}{ Arytenoid cartilage $(n=110)$} \\
\hline MRI & 8 & 91 & 1 & 10 & $89(52-100)$ & $91(83-95)$ & $44(22-69)$ & $99(94-100)$ \\
\hline Dual-energy CT & 6 & 98 & 3 & 3 & $67(30-93)$ & $97(92-100)$ & $67(30-93)$ & 97 (92-99) \\
\hline$P$ value ${ }^{c}$ & & & & & .43 & .09 & & \\
\hline \multicolumn{9}{|c|}{ All cartilage $(n=220)$} \\
\hline MRI & 35 & 155 & 1 & 29 & $97(85-100)$ & $84(78-89)$ & $55(42-67)$ & 99 (96-100) \\
\hline Dual-energy CT & 29 & 180 & 7 & 4 & $81(64-92)$ & $98(95-99)$ & 88 (72-97) & $96(92-98)$ \\
\hline$P$ value ${ }^{c}$ & & & & & .16 & $<.004$ & & \\
\hline
\end{tabular}

Note:-TP indicates true positive; TN, true negative; FP, false positive; FN, false negative; PPV, positive predictive value; NPV, negative predictive value.

${ }^{a}$ Numbers in parentheses are $95 \%$ confidence intervals. Negative findings for cartilage invasion are scores 1 and 2; positive findings are scores 3-5.

${ }^{b}$ As determined with the McNemar test.

${ }^{c}$ According to the generalized linear mixed model that accounted for the multiple observations within patients.

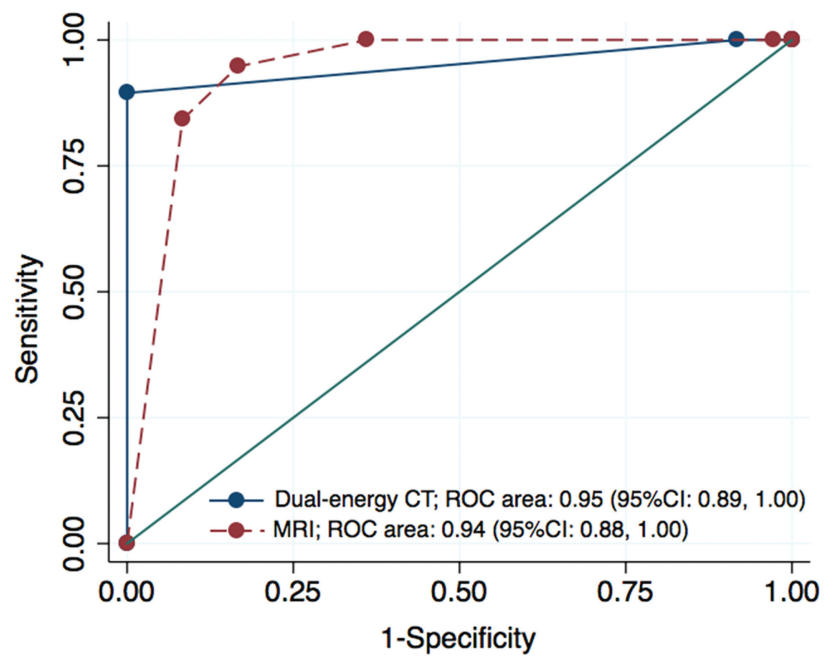

FIG 2. Graph shows 2 crossing ROC curves and corresponding AUCs in the prediction of thyroid cartilage invasion. There was no evidence of differences in the average areas under the ROC curve between MR imaging and dual-energy CT ( 0.938 versus 0.952 , respectively; $P=.70)$.

duction of overestimation for thyroid cartilage is particularly important for treatment-related decision-making compared with the reduction of underestimation for cartilage invasion; therefore, we believe that dual-energy CT may potentially assist in patient management.

MR imaging has a high sensitivity and high negative predictive value for detecting cartilage invasion compared with conventional CT. ${ }^{10}$ However, inflammatory changes in cartilage often resemble cartilage invasion, and high false-positive rates remain an issue. ${ }^{10,12}$ In our study, MR imaging demonstrated false-positive findings in areas of ossified cartilage with inflammatory change, and the higher sensitivity on MR imaging seems to be at the expense of specificity. Cortical bone and ossified cartilage are difficult to identify on MR imaging because of a lack of signal from bone. Therefore, MR imaging may be challenging for the evaluation of cortical bone changes, such as erosion or lysis. In addition, in patients with advanced SCCs, MR imaging seems to be prone to motion artifacts because of the relatively long scan times, which can render images nondiagnostic. In our study, the image quality of MR imaging tended to have motion artifacts, especially in patients with locally advanced (T3-T4) SCCs, while dualenergy CT showed no artifacts. Recently, phased array surface coils were applied to the neck for reducing the motion artifacts and increasing the spatial resolution. ${ }^{33,34}$ However, this technique uses a small FOV, restricting the imaged area to the larynx.

Dual-energy CT can provide WA images, which are like conventional $120-\mathrm{kV}$ images, and additional IO images, both with high spatial resolution. ${ }^{27,35,36}$ However, IO images have the technical limitation that lesions in ossified cartilage cannot be clearly identified. ${ }^{14}$ In our study, we therefore used combined WA and IO images for the diagnoses of both ossified and nonossified cartilage. The lesions that include calcified structures needed to be evaluated on WA images first because on IO images, iodine distribution could be overestimated due to the presence of calcified structures. When the WA image did not show cartilage destruction, regardless of inflammatory changes in the fatty marrow, cartilage invasion was considered absent in this diagnostic algorithm. High specificity for dual-energy CT could be achieved because the WA and IO images depicted the precise shapes of ossified cartilages and iodine distribution in nonossified thyroid cartilages and bone marrow space at the same time, thus preventing the overestimation of invasion that occurred during diagnoses with MR imaging. Furthermore, there is the potential for misclassification in the presence of artifacts that makes correlation and interpretation in conjunction with WA imperative.

There are limitations in our study. First, the difference between the signal intensities of the tumor adjacent to the cartilage and the nonossified cartilage was assessed visually without quantitative measurement of iodine concentrations. Therefore, the potentially confounding effect of interreader error, including display settings, may be present. In addition, there are other dualenergy CT approaches for evaluation of nonossified thyroid cartilage or head and neck squamous cell carcinoma in general that could potentially increase accuracy. These need to be evaluated in a future study. Second, because of the sample-size limitation, the 
statistical analysis was limited to the whole sample and each subgroup of primary tumor could not be analyzed. Besides, the small sample size may mask the lack of statistical difference in the sensitivity. Further studies with a larger number of patients are needed to provide further evidence for clinical relevance. Third, this study included only a small number of early-stage cases with no evaluation of their prognosis. If dual-energy CT promotes a more frequent selection of laryngeal-preserving therapy, it will be essential to evaluate long-term prognosis through collection of data from additional cases and to test the validity of this approach for each subset of tumors in a future prospective study.

\section{CONCLUSIONS}

The specificity of dual-energy CT is higher than that of MR imaging for the evaluation of cartilage invasion by laryngeal and hypopharyngeal SCCs for all cartilage combined and for thyroid cartilage alone. Dual-energy CT can prevent the overestimation of cartilage invasion that may occur due to inflammatory changes by using appropriate diagnostic criteria on WA images and IO images for ossified and nonossified cartilage. Although subtle tumor invasion into the ossified cartilage may be missed by dual-energy CT, it may add value by avoiding overestimation of cartilage invasion and may help in promoting laryngeal preservation approaches for laryngeal and hypopharyngeal SCCs.

Disclosures: Hirofumi Kuno-RELATED: Other: Siemens Healthcare K.K., Comments: One of the authors (K.O.) is an employee of Siemens Healthcare K.K., and Siemens Healthcare K.K. provided a license for iterative CT reconstruction (SAFIRE) to the National Cancer Center Hospital East for this study. Authors who are not employees of Siemens Healthcare monitored and had control of inclusion of any data and information submitted for publication*; UNRELATED: Grants/Grants Pending: Grant-in-Aid for Young Scientists (B) KAKEN (\#26861033). Katharina Otani-OTHER RELATIONSHIPS: Employee of Siemens Healthcare K.K. Osamu Sakai-UNRELATED: Consultancy: Guerbet, Boston Imaging Core Lab; Payment for Lectures Including Service on Speakers Bureaus: Bracco Diagnostics, Eisai. Masahiko KusumotoUNRELATED: Payment for Lectures Including Service on Speakers Bureaus: Ono Pharmacy, MSD, AstraZeneca. *Money paid to the institution.

\section{REFERENCES}

1. Baugnon KL, Beitler JJ. Pitfalls in the staging of cancer of the laryngeal squamous cell carcinoma. Neuroimaging Clin N Am 2013;23: 81-105 CrossRef Medline

2. Hermans R. Staging of laryngeal and hypopharyngeal cancer: value of imaging studies. Eur Radiol 2006;16:2386-400 CrossRef Medline

3. Pfister DG, Laurie SA, Weinstein GS, et al; American Society of Clinical Oncology. American Society of Clinical Oncology clinical practice guideline for the use of larynx-preservation strategies in the treatment of laryngeal cancer. J Clin Oncol 2006;24:3693-704 CrossRef Medline

4. Forastiere AA, Goepfert H, Maor M, et al. Concurrent chemotherapy and radiotherapy for organ preservation in advanced laryngeal cancer. N Engl J Med 2003;349:2091-98 CrossRef Medline

5. Hoffman HT, Porter K, Karnell LH, et al. Laryngeal cancer in the United States: changes in demographics, patterns of care, and survival. Laryngoscope 2006;116:1-13 Medline

6. Choi YS, Park SG, Song EK, et al; Korean South West Oncology Group (KSWOG) Investigators. Comparison of the therapeutic effects of total laryngectomy and a larynx-preservation approach in patients with T4a laryngeal cancer and thyroid cartilage invasion: a multicenter retrospective review. Head Neck 2016;38:1271-27 CrossRef Medline

7. Knab BR, Salama JK, Solanki A, et al. Functional organ preservation with definitive chemoradiotherapy for T4 laryngeal squamous cell carcinoma. Ann Oncol 2008;19:1650-54 CrossRef Medline
8. Lefebvre JL, Ang KK. Larynx preservation clinical trial design: key issues and recommendations - a consensus panel summary. Head Neck 2009;31:429-41 CrossRef Medline

9. Li B, Bobinski M, Gandour-Edwards R, et al. Overstaging of cartilage invasion by multidetector CT scan for laryngeal cancer and its potential effect on the use of organ preservation with chemoradiation. Br J Radiol 2011;84:64-69 CrossRef Medline

10. Becker M, Zbaren P, Laeng H, et al. Neoplastic invasion of the laryngeal cartilage: comparison of MR imaging and CT with histopathologic correlation. Radiology 1995;194:661-69 CrossRef Medline

11. Kuno H, Onaya H, Fujii S, et al. Primary staging of laryngeal and hypopharyngeal cancer: CT, MR imaging and dual-energy CT. Eur J Radiol 2014;83:e23-35 CrossRef Medline

12. Becker M, Zbären P, Casselman JW, et al. Neoplastic invasion of laryngeal cartilage: reassessment of criteria for diagnosis at MR imaging. Radiology 2008;249:551-59 CrossRef Medline

13. Dadfar N, Seyyedi M, Forghani R, et al. Computed tomography appearance of normal nonossified thyroid cartilage: implication for tumor invasion diagnosis. J Comput Assist Tomogr 2015;39:240-43 CrossRef Medline

14. Kuno H, Onaya H, Iwata R, et al. Evaluation of cartilage invasion by laryngeal and hypopharyngeal squamous cell carcinoma with dualenergy CT. Radiology 2012;265:488-96 CrossRef Medline

15. Tawfik AM, Kerl JM, Bauer RW, et al. Dual-energy CT of head and neck cancer: average weighting of low- and high-voltage acquisitions to improve lesion delineation and image quality-initial clinical experience. Invest Radiol 2012;47:306-11 CrossRef Medline

16. Vogl TJ, Schulz B, Bauer RW, et al. Dual-energy CT applications in head and neck imaging. AJR Am J Roentgenol 2012;199:S34-39 CrossRef Medline

17. Wichmann JL, Nöske EM, Kraft J, et al. Virtual monoenergetic dualenergy computed tomography: optimization of kiloelectron volt settings in head and neck cancer. Invest Radiol 2014;49:735-41 CrossRef Medline

18. Albrecht MH, Scholtz JE, Kraft J, et al. Assessment of an advanced monoenergetic reconstruction technique in dual-energy computed tomography of head and neck cancer. Eur Radiol 2015;25:2493-501 CrossRef Medline

19. Forghani R, Levental M, Gupta R, et al. Different spectral Hounsfield unit curve and high-energy virtual monochromatic image characteristics of squamous cell carcinoma compared with nonossified thyroid cartilage. AJNR Am J Neuroradiol 2015; 36:1194-200 CrossRef Medline

20. Lam S, Gupta R, Levental M, et al. Optimal virtual monochromatic images for evaluation of normal tissues and head and neck cancer using dual-energy CT. AJNR Am J Neuroradiol 2015;36:1518-24 CrossRef Medline

21. Scholtz JE, Kaup M, Kraft J, et al. Objective and subjective image quality of primary and recurrent squamous cell carcinoma on head and neck low-tube-voltage $\mathbf{8 0 - k V p}$ computed tomography. Neuroradiology 2015;57:645-51 CrossRef Medline

22. Lam S, Gupta R, Kelly H, et al. Multiparametric evaluation of head and neck squamous cell carcinoma using a single-source dual-energy CT with fast $\mathrm{kVp}$ switching: state of the art. Cancers (Basel) 2015;7:2201-16 CrossRef Medline

23. Hermans R, Vander Goten A, Baert AL. Image interpretation in CT of laryngeal carcinoma: a study on intra- and interobserver reproducibility. Eur Radiol 1997;7:1086-90 CrossRef Medline

24. Pache G, Krauss B, Strohm P, et al. Dual-energy CT virtual noncalcium technique: detecting posttraumatic bone marrow lesionsfeasibility study. Radiology 2010;256:617 CrossRef Medline

25. Joe E, Kim SH, Lee KB, et al. Feasibility and accuracy of dual-source dual-energy CT for noninvasive determination of hepatic iron accumulation. Radiology 2012;262:126-35 CrossRef Medline

26. Postma AA, Hofman PA, Stadler AA, et al. Dual-energy CT of the brain and intracranial vessels. AJR Am J Roentgenol 2012;199: S26-33 CrossRef Medline

27. Flohr TG, McCollough CH, Bruder H, et al. First performance eval- 
uation of a dual-source CT (DSCT) system. Eur Radiol 2006;16: 256-68 CrossRef Medline

28. Becker M, Zbären P, Delavelle J, et al. Neoplastic invasion of the laryngeal cartilage: reassessment of criteria for diagnosis at CT. Radiology 1997;203:521-32 CrossRef Medline

29. Beitler JJ, Muller S, Grist WJ, et al. Prognostic accuracy of computed tomography findings for patients with laryngeal cancer undergoing laryngectomy. J Clin Oncol 2010;28:2318-22 CrossRef Medline

30. Gupta R, Phan CM, Leidecker C, et al. Evaluation of dual-energy CT for differentiating intracerebral hemorrhage from iodinated contrast material staining. Radiology 2010;257:205-11 CrossRef Medline

31. Sim J, Wright CC. The kappa statistic in reliability studies: use, interpretation, and sample size requirements. Phys Ther 2005;85: 257-68 Medline
32. Obuchowski NA. Receiver operating characteristic curves and their use in radiology. Radiology 2003;229:3-8 CrossRef Medline

33. Allegra E, Ferrise P, Trapasso S, et al. Early glottic cancer: role of MRI in the preoperative staging. Biomed Res Int 2014;2014:890385 CrossRef Medline

34. Ravanelli M, Farina D, Rizzardi P, et al. MR with surface coils in the follow-up after endoscopic laser resection for glottic squamous cell carcinoma: feasibility and diagnostic accuracy. Neuroradiology 2013;55:225-32 CrossRef Medline

35. Graser A, Johnson TRC, Chandarana H, et al. Dual energy CT: preliminary observations and potential clinical applications in the abdomen. Eur Radiol 2009;19:13-23 CrossRef Medline

36. Johnson TR, Krauss B, Sedlmair M, et al. Material differentiation by dual energy CT: initial experience. Eur Radiol 2007;17:1510-17 CrossRef Medline 